12. Vypanasenko, S.I., \& Dreshpak, N.S. (2013). The features of energy efficiency measurement and control of production processes. Energy Efficiency Improvement of Geotechnical Systems, 1, 71-78.

13. Dreshpak, N.S., Dreshpak, O.S., \& Vypanasenko, S.I. (2021). Specific norms of energy consumption in the task of controlling the efficiency of its use. "Electrical engineering and electric power engineering", 3, 31-39.

https://doi.org/10.31713/m1108

\title{
DESIGN-INDUCED OPERATIONAL CHANGES OF STRESS-STRAIN STATE IN FLAT RUBBER-CABLE TRACTIVE ELEMENT OF HOISTING AND TRANSPORTING MACHINE
}

Kolosov D.L.

Dnipro University of Technology, Dnipro, Dr. Sc. (Tech.), Associate Prof., Head of Department of Structural, Theoretical and Applied Mechanics, Ukraine

Samusia V.I.

Dnipro University of Technology, Dnipro, Dr. Sc. (Tech.), Associate Prof., Head of Department of Mining Mechanics, Ukraine

\section{Bilous O.I.}

Dniprovsk State Technical University, Kamianske, Cand. Sc. (Tech.), Associate Prof., Associate Prof. of Department of Mechanical Engineering, Ukraine

\section{Tantsura H.I.}

Dniprovsk State Technical University, Kamianske, Cand. Sc. (Tech.), Associate Prof., Associate Prof. of Department of Mechanical Engineering, Ukraine

\section{Onyshchenko S.V.}

Dnipro University of Technology, Dnipro, Cand. Sc. (Tech.), Associate Prof. of Department of Structural, Theoretical and Applied Mechanics, Ukraine

Abstract.

Main indicators of a stress-strain state (SSS) of a rubber-cable belt, the rubbercable rope for a random placement pattern and the size of the part with broken cables, including partially removed cables, with cable breakages and their random 
amount, different conditions of belt interaction in its cross-sections of connection to structural elements of a conveyor and a lifting machine to ensure control of its tractive ability during the life cycle. Performed researches clarify the idea of the interaction mechanism of reinforcing elements in composite materials of layered structure with hard and soft layers. Obtained results can be used for development and justification of a unified technology of creation and engineering support of operation of lifting and transporting machines with flat tractive-bearing elements with increased life-span, level of efficiency and operational safety in systems of extraction, transportation and processing of minerals.

\section{Introduction}

Lifting and transporting complexes are the most important link in the chain of mineral extraction. The economic efficiency and social stability of a mining complex $[1,2]$ as a whole depend on an uninterrupted and safe operation of lifting and transporting complexes [3]. Effective and trouble-free operation of lifting and transporting machines, including ones with flat tractive-bearing elements, can be provided by creation and realization of bases of scientifically justified engineering support throughout the life cycle their operation.

Rubber-cable flat belts (RCB) are used in lifting and transporting engineering. They perform the function of tensile force transmission. Transported material is on the conveyor belt. Conveyor belts have a closed form. Rubber-cable and rubber-fabric flat belts have a system of tractive elements located along its longitudinal axis. Reinforcing elements are placed with the same spacing along the belt width. An elastic shell provides the structural integrity of tractive elements into which they are packed. Tractive elements are broken during operation. Belts can be used in sifting machines, in conveyors for partial water discharge during the transportation of moist lumpy mass. To do this, it is enough to make holes in a belt.

Formation of artificial holes and breakage of tractive elements during the belt operation lead to a change in their structure. Changing the structure leads to a change in a mechanism of interaction of reinforcing elements. This change leads to a redistribution of loads between the reinforcing elements, occurrence of tangential stresses in a belt shell and, eventually, to a reduction of tractive ability of a flat belt (rope).

Reduction of a tractive ability can lead to unpredictable consequences. The variety of possible patterns of changing the structure of a belt reinforcement due to a need to adapt the belt to the needs of technological process, design features of the machine, the breakage 
of its elements during the operation process requires the solution of the actual task - the development of a single method for determining the stress-strain state of a flat elastic belt reinforced with longitudinal tractive elements.

\section{State of Question and Statement of Research Problem}

Conveyors of various designs, including steeply inclined [4], with a suspending belt [5, 6], tubular [7], are designed for transportation of considerable amounts of material. They are mostly equipped with rubber-cable belts. Belt cables can have ruptures of continuity. So the continuity of tractive elements is ruptured in butt-joint connections. The connections are performed in order to obtain a belt of considerable length, to provide it with a closed form on the conveyor. The ruptures of continuity of cables lead to a decrease in endurance and strength of belts [8,9], in particular in butt-joint connections [10]. Properties of butt-joint connections of rubber-cable conveyor belts that were in operation are investigated in [11]. The holes can be made in belts [12]. Cables can break during operation of belts. Tractive ability and operational reliability of belts [13] and their butt-joint connections [14] are reduced during operation.

Reduction of a tractive ability of ropes and belts can lead to unpredictable consequences. Failure of conveyors due to a failure of belts and support rollers in the air-salt medium reaches $47 \%$ [15]. The method for determining the value of a safe load on a belt with defects is justified in [16]. It is based on empirical dependency and does not involve the case of removal of cable parts. The issue of determining the reduced mechanical characteristics of composite materials reinforced by a system of regularly arranged, parallel reinforcing elements of nonsignificant rigidity is depicted in [17]. The effect of the geometric parameters of the rubber-cable belt on its stress-strain state in the interaction with clamping elements on the driving drum winder is investigated in [18].

Paper [19] suggests an algorithm for determining the stress-strain state of a flat rubber-cable rope with one broken cable, considering the deviation of a drum generatrix from a straight line. The possibility of consideration of design parameters of a machine by means of assigning certain boundary conditions of loading of a rubber belt (rope) is displayed.

Known algorithms for determining a stress-strain state of a flat rubber-cable conveyor belt, a rope of a lifting machine can't be used to determine a tractive ability of a belt with various cable disturb- 
ances, including belts with systematically located holes.

Rubber-cable belts consist of a system of parallel cables, located along the belt in the same plane. Rubber-fabric belts have a system of parallel threads located in several planes. In a case of longitudinal loading, including the case of formation of through holes, the threads are located in parallel planes and deform in the same manner. Mechanisms of deformation of rubber-cable and rubber-fabric belts can be considered close. Consider a rubber-cable belt, which is used as a flat rope. Along the belt width, the cables are located with a constant spacing and packed into an elastic shell. Longitudinal deformations of cables are much larger than their deformations in the plane of a belt. The elastic shell takes almost only shear stress. Deformations of cables are insignificant in the plane of a belt. Neglect the influence of a difference between the twisting angles of adjacent cables in the plane of a belt to the values of tangent stresses in the elastic material. The specified allows considering rubber-cable belt as a composite material of regular layered structure with rigid and soft layers.

Breakages of a tractive core of the belt, made artificially or those that occurred during the operation rupture the regular structure of the belt. Belts with random breakages of reinforcing elements partially lose a regularity of placement of reinforcing elements. The latter case can be considered as a separate case of a regular change in the belt structure. Belts interact with elements of conveyors of various designs.

Mechanical properties of the belt, including its strength, depend on the amount, relative placement, mechanical properties of reinforcing elements and shell material, and the character of distribution of forces between tractive elements. The task of determining the distribution of forces between tractive elements is statically indeterminate. The distribution of forces depends on a character of interaction of the belt with individual structural elements of the conveyor. The formation of holes in such belts is connected to the partial removal of reinforcing elements and the reduction of its tractive ability.

The task is to create an algorithm for determining the main indicators of the stress-strain state of a rubber-cable belt and a rubber-cable rope for a random placement pattern and the size of the part with broken cables, including partially removed cables, with cable breakages and their random amount, different conditions of belt interaction in its cross-sections of connection to structural elements of a conveyor and 
a lifting machine to ensure control of its tractive ability during the life cycle.

\section{Belt with Regular Ruptures of Continuity of Cable Groups}

The main tractive elements of a belt and a rope are cables. A rubber-cable rope of the lifting machine and a rubber-cable belt are called a belt henceforth. Belt cables consist of strands. Strands consist of wires. Wires in strands as well as strands in cables are twisted. This structure leads to a twisting moment of a cable when it is loaded with longitudinal force. In order to balance the twisting, the belt structure has an even number of cables. Correspondingly, the number of removed tractive elements in a special hole in the belt should be even. The number of broken cables in a general case can be random.

The lengths of conveyors on which rubber-cable belts are installed are significant. This allows disregarding the circular (closed) shape of a belt. Consider a belt in which two opposite edges are attached to structural elements of a conveyor. The load character of a belt on the conveyor is determined by its structure. Features of interaction of a belt with conveyor drums can be defined by boundary conditions.

Considering the upper-mentioned and in order to obtain a solution for a general case, consider the rubber-cable belt with a length $l_{1}+l_{2}$. The belt has $M+N$ cables. Broken (removed) group of $N$ cables out of these $M+N$ cables is located on the part of a belt with length $l_{2}$. The group is located at the edge of the belt. It is loaded with a tensile force $P$ at random boundary conditions. Cables have a considerable tensile rigidity. The main deformation of the elastic shell is its shear between the cables.

Cable displacements in a belt, as a layered structure with hard and soft layers can be determined by dependency [19], which is obtained from the condition of cables equilibrium on a belt part of a constant regular structure

$$
u(i, x)=\sum_{n=1}^{M-1}\left(\frac{A_{n} e^{\beta_{n} x}+B_{n} e^{-\beta_{n} x}}{\beta_{n}}\right) \cos \left(\mu_{n}(i-0.5)\right)+\frac{P x}{Z E F}+c,
$$

where $u(i, x)$ - displacement of a cable $i(1 \leq i \leq Z)$ in a crosssection $x ; A_{n}, B_{n}, c$ - unknown constants;

$$
\mu_{n}=\frac{\pi n}{Z} ; \beta_{n}=\sqrt{\frac{2 G k_{G}}{(t-d) E F}\left(1-\cos \left(\mu_{n}(i-0.5)\right)\right)} ; Z, d, t \text { - amount, }
$$


diameter and spacing of cable placement in a belt; $E, F$ - reduced tensile rigidity of a cable and its cross-sectional area; $G$ - reduced shear modulus of an elastic shell of the belt; $k_{G}$ - the coefficient that considers the influence of the elastic shell material shape located between the cables.

Rupture of integrity of cables leads to a rupture of a belt structure and eliminates the possibility of using (1). Use the method of crosssections. Cut the belt into two parts along the location of a crosssection of cable breakage (along the cross-section of their amount change in parts of a belt). Within each of these parts of a belt its structure is unchanged. Expression (1) is applicable for each part. For the first part the amount of cables $Z$ equals $M+N$. For the second one $Z=M$. The stress-strain state of the entire belt can be determined if the condition of joint deformation of both parts and the condition of loading of belt boundaries is ensured.

Give belt parts numbers 1 and 2 . The numbers are written in the indexes of parameters related to the first and second parts of the belt. The beginning of the $x$-axis is located on the common boundary of both parts. Formulate boundary conditions and condition of joint deformation of belt parts.

Assume that deformations at the belt edges are defined by func$f_{1}(i)=\sum_{n=1}^{M+N} F_{1, n} \cos \left(\mu_{1, n}(i-0.5)\right)$
$f_{2}(i) \sum_{n=1}^{M} F_{2, n} \cos \left(\mu_{2, n}(i-0.5)\right)$. Formulate boundary conditions: in a cross-section

$$
x=-l_{2} \quad u_{2, i}=f_{2}(i), \sum_{i=1}^{M} p_{i}=P
$$

and when

$$
x=l_{1} \quad u_{1, i}=f_{1}(i), \quad \sum_{i=1}^{M+N} p_{i}=P,
$$

where $p_{i}$ - tensile force of cable $i$.

Conditions of joint deformation of parts (in a cross-section $x=0$ ) 


$$
\begin{gathered}
u_{1, i}=u_{2, i}(1 \leq i \leq M), \\
p_{1, i}=p_{2, i} \quad(1 \leq i \leq M), \\
p_{1, i}=0(M<i \leq N+M) .
\end{gathered}
$$

From the boundary conditions the following dependencies are formulated

$$
\begin{aligned}
& A_{1, n}=\left(F_{1, n}-B_{1, n} e^{-\beta_{1, n} l_{1}}\right) e^{-\beta_{1, n} l_{1}}, \\
& A_{2, n}=\left(F_{2, n}-B_{2, n} e^{\beta_{2, n} l_{2}}\right) e^{\beta_{2, n} l_{2}} .
\end{aligned}
$$

The obtained ratios allow reducing the amount of unknowns in expressions for displacements and internal loads of cables in a belt

$$
\begin{gathered}
u_{1}(i, x)=\sum_{n=1}^{M+N-1}\left(F_{1, n} e^{-\beta_{1, n} l_{1}} e^{\beta_{1, n} x}+B_{1, n}\left(e^{-\beta_{1, n} x}-e^{-2 \beta_{1, n} l_{1}} e^{\beta_{1, n} x}\right)\right) \times \\
\quad \times \frac{\cos \left(\mu_{1, n}(i-0.5)\right)}{\beta_{1, n}}+\frac{P x}{(M+N) E F}+c_{1}, \\
u_{2}(i, x)=\sum_{n=1}^{M-1}\left(F_{2, n} e^{\left.\beta_{2, n} l_{2} e^{\beta_{2, n} x}+B_{2, n}\left(e^{-\beta_{2, n} x}-e^{2 \beta_{2, n} l_{2}} e^{\beta_{2, n} x}\right)\right) \times}\right. \\
\times \frac{\cos \left(\mu_{2, n}(i-0.5)\right)}{\beta_{2, n}}+\frac{P x}{M E F}+c_{2} .
\end{gathered}
$$

Coefficient $c_{1}$ is assumed zero. Then $c_{2}=u_{1}(i, 0),(1 \leq i \leq M)$

Expressions of displacement (6), (7) include unknown vectors of displacements with values $N+M-1$ and $M-1$. In order to reduce the number of vectors of unknown constants, the first $(M-1)$ constants of a vector $B_{1, m}$ are expressed through unknown constant $B_{2, n}$. The numbers of cables are considered as a discrete coordinate axis. Internal forces occurring in cables of the first part are written in a form of Fourier series on a segment $(0<i<M+N+1)$ 


$$
\begin{aligned}
& p_{1}(i, x)=\frac{2 E F}{M+N} \times \\
& \times \sum_{m=1}^{M+N-1}\left[\begin{array}{l}
\sum_{n=1}^{M-1}\left(\times \sum_{j=1}^{M} \cos \left(\mu_{2, n}(j-0.5)\right) \cos \left(\mu_{1, m}(j-0.5)\right)\right. \\
\beta_{2, n} l_{2}+B_{2, n}\left(1-e^{2 \beta_{2, n} l_{2}}\right) \times \\
+\sum_{j=M+1}^{M+N} B_{1, j} \cos \left(\mu_{1, m}(j-0.5)\right)
\end{array}\right] \times \\
& \times\left(\frac{e^{-\beta_{1, m} l_{1}} e^{\beta_{1, m} x}+e^{-\beta_{1, m} x}}{e^{-\beta_{1, m} l_{1}}-1}\right) \cos \left(\mu_{1, m}(i-0.5)\right)+\frac{P}{M+N} .
\end{aligned}
$$

In the resulting expression, the sum of members with unknown values of a vector of constant $B_{2, n}$, reproduces the condition of equality of loads of cables belonging to the first and second parts in the cross-section $x=0$. The sum of members of an unknown vector $B_{1, j}(M \leq j \leq M+N-1)$ describes the distribution of forces between cables of the first part, the ends of which are located in the same cross-section.

The internal loading forces of cables of the second part are determined from (7) by Hooke's law

$$
\begin{gathered}
p_{2}(i, x)= \\
=\sum_{n=1}^{M-1}\left(F_{2, n} e^{\beta_{2, n} l_{2}} e^{\beta_{2, n} x}-B_{2, n}\left(e^{-\beta_{2, n} x}-e^{2 \beta_{2, n} l_{2}} e^{\beta_{2, n} x}\right)\right) \times \\
\times \cos \left(\mu_{2, n}(i-0.5)\right) E F+\frac{P}{M} .
\end{gathered}
$$

Accepted forms of solutions ensure the implementation of condition of equality of forces in terms of joint deformation of parts of an unbroken cable belonging to both parts of the belt (4). The condition of equality of displacements of cables with numbers $1 \leq i \leq M$ in a cross-section $x=0$ and the condition where internal forces in cables are equal to zero, that were determined in conditions of joint deformation of belt parts (4), allow creating a system of algebraic equations of order $M+N-1$. The belt has $M+N$ cables in the first part. 
Note that the expressions of internal forces (8) and (9) have two components. The first depends on a cable number and a value of coordinate $x$. The second is a constant value. The sum of forces defined only by the first components equals zero. This allows not defining the condition of load absence for one of the cables with a number greater than $M$. The specified condition is executed automatically. Order of the system of equations $M+N-1$ is sufficient to determine all unknown constants.

Known values of constants allow determining the distribution of forces between cables and their displacements. The displacements allow determining the tangential forces transmitted by the elastic shell located between the cables. Tangential forces are proportional to displacement angles of elastic shell material. Tangents of displacement angles are determined by the difference in displacements of adjacent cables related to the distance between them.

Belt strength is determined by maximum loads of cables and forces transmitted by an elastic shell between them. The maximum loaded cable is adjacent to the broken one in the breakage crosssection. Expression (8) makes it possible to find this maximum force

$$
\begin{aligned}
p_{\max }= & \sum_{n=1}^{M-1}\left(F_{2, n} e^{\beta_{2, n} l_{2}}-B_{2, n}\left(1-e^{2 \beta_{2, n} l_{2}}\right)\right) \times \\
& \times \cos \left(\frac{\pi n}{2 M}\right)(-1)^{n} E F+\frac{P}{M} .
\end{aligned}
$$

The maximum tangential forces are transmitted by an elastic shell in the part at the end of a broken cable adjacent to the unbroken one. They correspond to the maximum displacement angle. The tangent of maximum displacement angle is determined by the dependency

$$
\begin{gathered}
\alpha_{\max }=\sum_{n=1}^{M+N-1}\left(F_{1, n} e^{-\beta_{1, n} l_{1}}+B_{1, n}\left(1-e^{-2 \beta_{1, n} l_{1}}\right)\right) \times \\
\times \frac{\cos \left(\mu_{1, n}\left(M-\frac{1}{2}\right)\right)-\cos \left(\mu_{1, n}\left(M+\frac{1}{2}\right)\right)}{\beta_{1, n}} .
\end{gathered}
$$

A condition, that the group of adjacent cables at the edge of the belt is broken (removed) is accepted above. The results obtained are also acceptable in a case when a group of broken cables is symmetrically 
located along the longitudinal axis of the belt. This is due to the fact that in the symmetry plane there are no tangential stresses in a rubber shell, as they do not occur on the belt side. The difference is in the fact that in a case of breakage of a group of cables in the middle of the belt the number of broken $N$ and unbroken $M$ cables in the expressions above should be taken twice as small as the actual ones. Thus, (10) and (11) are also acceptable for the case when broken cables are located in the middle of a belt. Locations of breakages at the edge of a belt and symmetrically to its axis are extreme cases. Correspondingly, indicators of a stress-strain state for other cases do not exceed the indicators specified for two cases.

Obtained expressions of maximum internal forces taken by cables and the maximum values of tangents of displacement angles determine the maximum values of indicators of a stressstrained state of a rubber-cable belt.

Consider using the offered method on an example of a belt type RCB-3150. In order to remove moisture from washed agricultural products, holes are made in a conveyor belt with a removal of parts of longitudinal tractive elements. Tractive elements, in general, may not be metallic. But their longitudinal deformations greatly exceed their normal displacements in the belt plane. Elastic shell transfers shear stresses. Uniform moisture drainage, regardless of the location of holes in a belt, that moves and has a closed form, can be provided with a uniform hole placement along the length and width of the belt.

Assume that the holes have dimensions $l_{2} \times b$. Place $x$-axis along the belt. The start of axis is on the boundary of a random row of holes (Fig. 1).

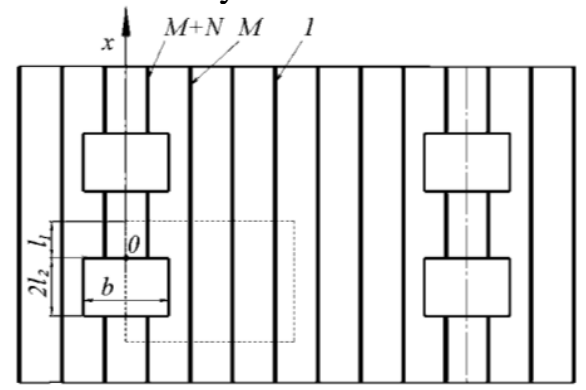

Fig. 1. Diagram of placement of tractive elements and holes with dimensions $l_{2} \times b$ on a belt

Give numbers to cables from one to $M+N$. Value $M+N$ deter- 
mines the amount of cables in a symmetrical part of a repeating element of the belt. Value of the amount of cables $N$ depends on a hole width and a cable placement spacing and is determined by the formula $N=b / 2 t$. Cables are shown with thickened lines. Show the symmetrical part of a repeating belt element with a system of holes arranged with a constant spacing, along the belt and along its width using dash lines. The symmetry of the specified element leads to the following features of its deformation. Cross-sections in planes of symmetry, located normally to $x$-axis, don't curve. Displacements of reinforcing elements located symmetrically to the edge of the hole of parallel axis are equal. Tangential stresses do not occur in the rubber between specified tractive elements during the belt loading with tensile forces. There are no tangential stresses in the area near a hole.

In a part $x>0$ the amount of tractive elements is $M+N$. In a part $x<0-M$. As performed before, split the belt into two parts by a cross-section $x=0$. Indicate them with numbers 1 and 2 . The numbers are used as indexes to indicate the values of the corresponding belt part.

Formulate boundary conditions

a) in a cross-section

$$
x=l_{1} \quad u_{2 i}=u_{1, i \pm 1}, p_{1, i}=\frac{P}{M+N},
$$

b) in a cross-section

$$
x=\frac{l_{2}}{2} \quad u_{2 i}=u_{2, i \pm 1} .
$$

At boundary (12) we have $F_{1, n}=0(1 \leq n \leq M+N)$. From condition (13) $-A_{2, n}=0(1 \leq n \leq M)$.

Displacements and internal loading forces of cables are determined from (6)-(9) and appear as shown in (14)-(17).

Using Hooke's law in (8), determine displacements of cables of the first belt part.

Use the conditions of joint deformation of belt parts (4). Construct a system of algebraic equations of order $M+N-1$ (18). 


$$
\begin{aligned}
& u_{1}(i, x)=\sum_{n=1}^{M+N-1} B_{1, n}\left(e^{-\beta_{1, n} x}-e^{-2 \beta_{1, n} l_{1}} e^{\beta_{1, n} x}\right) \times \\
& \times \frac{\cos \left(\mu_{1, n}(i-0.5)\right)}{\beta_{1, n}}+\frac{P x}{(M+N) E F}, \\
& u_{2}(i, x)=\sum_{n=1}^{M-1} B_{2, n}\left(e^{-\beta_{2, n} x}-e^{2 \beta_{2, n} l_{2}} e^{\beta_{2, n} x}\right) \times \\
& \times \frac{\cos \left(\mu_{2, n}(i-0.5)\right)}{\beta_{2, n}}+\frac{P x}{M E F}+c_{2}, \\
& p_{1}(i, x)=\sum_{m=1}^{M+N-1}\left[\begin{array}{l}
\sum_{n=1}^{M} B_{2, n}\left(\frac{e^{-\beta_{2, n} l_{2}}-1}{\beta_{2, n}}\right) \times \\
\left.\times \sum_{j=1}^{M} \cos \left(\mu_{2, n}(j-0.5)\right) \cos \left(\mu_{1, m}(j-0.5)\right)+\right] \times \\
+\sum_{j=M+1}^{M+N} B_{1, j} \cos \left(\mu_{1, m}(j-0.5)\right)
\end{array}\right] \\
& \times\left(\frac{e^{-\beta_{1, m} l_{1}} e^{\beta_{1, m} x}+e^{-\beta_{1, m} x}}{e^{-\beta_{1, m} l_{1}}-1}\right) \beta_{1, m} \cos \left(\mu_{1, m}(i-0.5)\right) \frac{2 E F}{M+N}+\frac{P}{M+N} \text {, } \\
& p_{2}(i, x)=\sum_{n=1}^{M} B_{2, n}\left(e^{-\beta_{2, n} l_{1}} e^{\beta_{2, n} x}+e^{-\beta_{2, n} x}\right) \times \\
& \times \cos \left(\mu_{2, n}(i-0.5)\right) E F+\frac{P}{M} .
\end{aligned}
$$




$$
\left\{\begin{array}{c}
\sum_{n=1}^{M-1} B_{2, n}\left(\frac{e^{-\beta_{2, n} l_{2}}-1}{\beta_{2, n}}\right)\left(\begin{array}{l}
\sum_{j=1}^{M} \cos \left(\mu_{2, n}(j-0.5)\right) \sum_{m=1}^{M+N-1} \frac{2 \cos \left(\mu_{1, m}(i-0.5)\right)}{M+N} \times \\
\times \cos \left(\mu_{1, m}(j-0.5)\right)-\cos \left(\mu_{2, n}(i-0.5)\right)
\end{array}\right)+ \\
+\sum_{j=M+1}^{M+N} B_{1, j} \sum_{m=1}^{M+N-1} \cos \left(\mu_{1, m}(i-0.5)\right) \frac{2}{M+N} \cos \left(\mu_{1, m}(j-0.5)\right)=0, \\
\quad \sum_{n=1}^{M} B_{2, n}\left(\frac{e^{-\beta_{2, n} l_{2}}-1}{\beta_{2, n}}\right) \sum_{j=1}^{M} \cos \left(\mu_{2, n}(j-0.5)\right) \times \\
+\sum_{m=1}^{M+N-1} \cos \left(\mu_{1, m}(j-0.5)\right)\left(\frac{e^{-2 \beta_{1, m} l_{1}}+1}{e^{-2 \beta_{1, m} l_{1}}-1}\right) \beta_{1, m} \cos \left(\mu_{1, m}(i-0.5)\right)+ \\
+\sum_{j=M+1}^{M+N} B_{1, j} \sum_{m=1}^{M+N-1} \cos \left(\mu_{1, m}(j-0.5)\right)\left(\frac{e^{-2 \beta_{1, m} l_{1}}+1}{e^{-2 \beta_{1, m} l_{1}}-1}\right) \beta_{1, m} \cos \left(\mu_{1, m}(i-0.5)\right)= \\
(M+1 \leq i \leq M+N-1) .
\end{array}\right.
$$

The width of holes $b$ is proportional to the amount of tractive elements. Set their amount equal to two and four. Solve the system of equations for these conditions. Find unknown constants. Determine the internal tensile forces of belt cables, their displacements, tangents of displacement angles of elastic material of a belt shell.

The concept of stress concentration factor is used in engineering practice. Analogically, define the tensile load concentration factor of tractive elements. The load concentration factor is the internal tensile force of a tractive element related to their average value on a belt part with $M+N$ cables. The load concentration factor depends on a change of the amount of cables in the belt part and a character of redistribution of forces between them. Considering the specified, the value of load concentration factor is considered a product of a relative load concentration factor $k_{r}$ and the coefficient of change of the amount of cables in the belt part with a reduced amount of cables, namely

$$
k_{n}=\frac{M+N}{M} \text {. }
$$

Then 


$$
k=k_{r} k_{n} .
$$

Similarly, values of cable displacements, values of tangents of displacement angles are considered as products of relative displacements, relative tangents of shear angles of the elastic layer and the coefficient of change in the number of cables in the belt part with a reduced amount of them, namely:

$$
\begin{aligned}
& u=u_{r} k_{n}, \\
& \alpha=\alpha_{r} k_{n} .
\end{aligned}
$$

Fig. 2 shows the distribution of relative load concentration factors, displacements and tangents of shear angles for the case $l_{1}=1 \mathrm{~m}$, $l_{2}=1 \mathrm{~m}, M=7, N=3$, in the cross-section $x=0$.

According to the graphs, the load concentration factors, and the shear angles achieve maximum values for cables adjacent to the broken cables and in the elastic shell between the continuous and broken cables. This is a consequence of the maximum gradient of displacements of adjacent unbroken and broken cables. The value of the relative load concentration factor is 1.17 , and the load concentration factor is 1.671 .

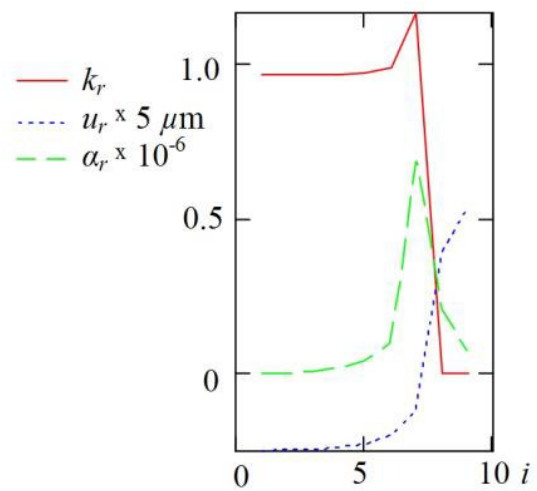

Fig. 2. Distribution of relative load concentration factors $\left(k_{r}\right)$, that occur in cables with numbers $i$, relative displacements $\left(u_{r}\right)$ and tangent of shear angles of elastic inter-cable layer $\left(\alpha_{r}\right)$ in a cross-section $x=0$

The stress-strain state of the belt under given boundary conditions depends on a number of factors such as size of belt parts, the amount of cables, including the broken. Fig. 3 shows the graphs of dependencies of maximum values of relative load concentration factors and relative shear angles tangents for the case $l_{2}=1 \mathrm{~mm}$. The length of the first one is $1 \mathrm{~mm}, 1,2,3, \ldots, 10 \mathrm{~m}$. The amount of cables $M=7, N=3$. 
Fig. 3 shows an increase in the load concentration factors and shears with increasing length of the first belt part if the length of the latter does not exceed $10 \mathrm{~m}$. This character of change of these parameters is a consequence of a joint effect of a local change in a belt structure and a limited belt deformation in the cross-section $x=l_{1}$.

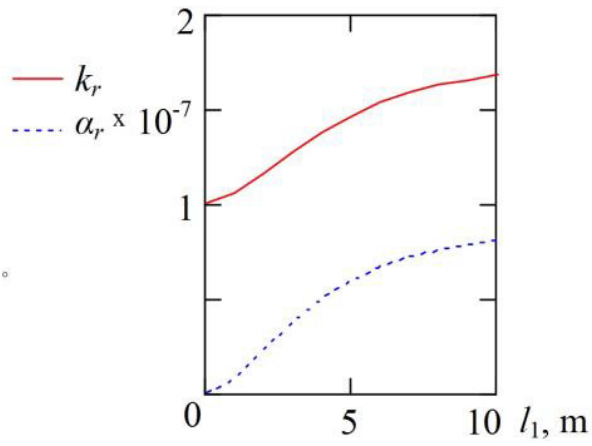

Fig. 3. Dependency of maximum values of relative internal load concentration factors $\left(k_{r}\right)$, that occur in cables and maximum shear angles tangents of elastic intercable layer $\left(\alpha_{r}\right)$ in a cross-section $x=0$ on the length of the first belt part and the length of the second part of $1 \mathrm{~mm}$

Consider the case of a constant length of the first part $l_{1}=10 \mathrm{~m}$. The length of the second one is $1 \mathrm{~mm}, 1,2,3, \ldots, 10 \mathrm{~m}$. According to St. Venant's principle, with increasing distance from the place of local perturbation of the stress-strain state, the latter approaches the uniform state. Belt cross-section that passes through the middle of the second part is the most distant from a cross-section of cable amount change. Considering this fact, determine the values of load concentration factors in the cross-sections $x=0$ and $x=l_{2}$, and maximum values of coefficients and shear (Fig. 4).

Shown calculation results indicate that the values of maximum stresses in the belt decrease with increasing length of the part where cables are partially removed. Maximum shears and load concentration factors decrease provided that the length of the belt part with removed cables does not exceed $5 \mathrm{~m}$. Maximum load concentration factors in the middle part are smaller than the corresponding concentration factors in the cross-section $x=0$. They decrease provided that the length of the belt part with removed cables does not exceed $11 \mathrm{~m}$. Tangential stresses in the middle section of the second belt part are absent. 


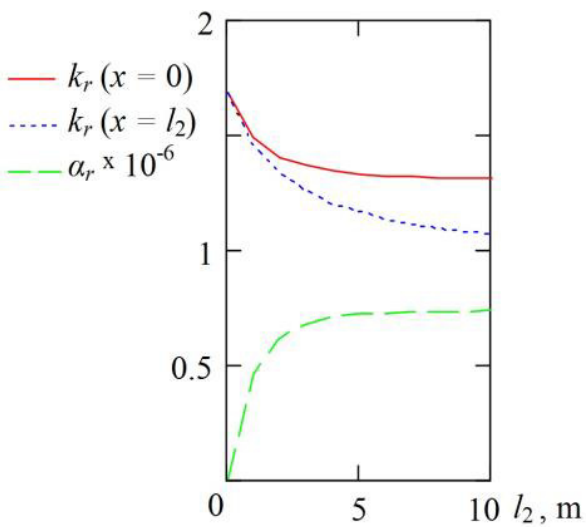

Fig. 4. Dependency of values of relative internal load concentration factors $\left(k_{r}=0\right)$ that occur in cables in a cross-section $x=0, k_{r}\left(x=l_{2}\right)$ in a cross-section $x=l_{2}$ and maximum shear angle tangents of elastic inter-cable layer $\left(\alpha_{r}\right)$ on the length of the second belt part and the length of the first part of $10 \mathrm{~m}$

The influence of the amount of partially removed cables on the stress state of the belt is shown in the Fig. 5.

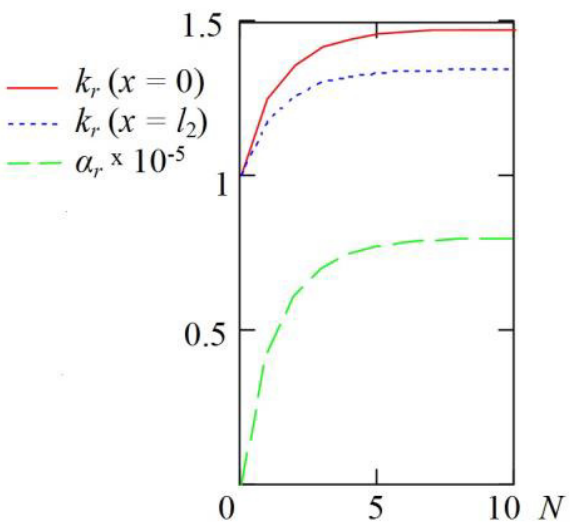

Fig. 5. Dependency of values of relative internal load concentration factors $k_{r}(x=0)$ that occur in cables in a cross-section $x=0, k_{r}\left(x=l_{2}\right)$ in a cross-section $x=l_{2}$ and maximum shear angle tangents of elastic inter-cable layer $\left(\alpha_{r}\right)$ on the amount of broken cables $N$

Fig. 5 shows the calculated values of concentration factors with the length of the second part of $3 \mathrm{~m}$, the first one $-10 \mathrm{~m}$. The amount of broken cables was chosen within the range from zero to 10 , and 
unbroken - 10. The graphs show that, in the absence of partially removed cables, the load concentration factors are equal to 1 . The mutual shear of cables is absent. The increase of the amount of partially removed cables leads to a nonlinear increase in the relative load concentration factors and shears, provided that the amount of partially removed cables does not exceed the number of unbroken. Absolute values of the load concentration factors and shear values in the case of quantitative excess of partially removed cables over the amount of unbroken cables practically linearly increase with an increase of the amount of partially removed cables.

Fig. 6 shows the values of concentration factors with the length of the second part of $3 \mathrm{~m}$, the first one - $10 \mathrm{~m}$. The amount of partially removed cables is 5, and unbroken - from 10 to 30 .

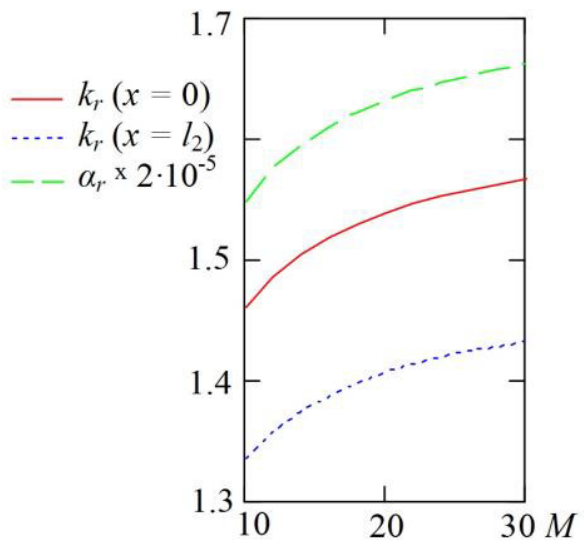

Fig. 6. Dependency of values of relative internal load concentration factors $k_{r}(x=0)$ that occur in cables in a cross-section $x=0, k_{r}\left(x=l_{2}\right)$ in a cross-section $x=l_{2}$ and maximum shear angle tangents of elastic inter-cable layer $\left(\alpha_{r}\right)$ on the amount of unbroken cables $M$

Graphs show that the increase of the amount of unbroken cables, like in the previous case, with the increase of the amount of partially removed cables, leads to a nonlinear increase of relative load concentration factors and shears. At the same time, the absolute values of the load concentration factors and shear values nonlinearly reduce with the increase of the amount of unbroken cables.

Compare the results for a belt with limited boundary deformations, if there are two parts in which the belt has unbroken cables 
and on a given length partially removed cables under different conditions. The most unevenly distributed forces between cables and the greatest tangential stresses in the elastic layers between cables occur when a length of the part with partially removed cables is approaching zero. The increase of a ratio of the amount of unbroken cables to their amount, which is removed on some length, leads to a nonlinear decrease in maximum tensile forces of cables and maximum shear of elastic layers located between cables.

The case of limited boundary deformations of a belt allows determining its stress state when it has a regularly variable structure. In the operation process of belts, there may be local, non-regular cases of belt breakage. Consider the case of a load of infinitely long belt, in the middle of which several cables are partially removed on a limited length. From (4)

$$
F_{1, n}=0
$$

Fig. 7 shows the distribution of relative load concentration factors, deformations and tangents of shear angles in the cross-section $x=0$ for a case similar to (Fig. 2), but for $l_{1} \rightarrow \infty$

According to the graphs in Fig. 7, the load concentration factors and the shear angles reach the maximum values for cables adjacent to broken cables and in the elastic shell between the unbroken and broken cables. This is a consequence of the maximum gradient of displacements of adjacent unbroken and broken cables. The value of the relative load concentration factor is 1.387 , and the load concentration factor is 1.982 .

Consider the dependency of the stress state of the belt on a length of the second belt part. The results are shown in Fig. 8 .

The given calculation results allow drawing the following conclusions. The values of maximum stresses in a belt with cables partially removed on a limited length depend on the amount of cables in the belt, the amount of partially removed cables, and the length of a part of their removal. So the minimum values of the load concentration factors are obtained in the case of removal of 3 cables from 10 in the belt with a length of the cable removal part of $1 \mathrm{~m}$. Minimum tangential stresses are realized for the length of the specified part of $0.5 \mathrm{~m}$. The presence of a minimum of maximum tensile forces and shear angles is the result of overlapping edge effects due to the change of 
the amount of cables on boundaries of the part of partial cable removal. This effect occurs with any amount of removed cables and their total amount in the belt.
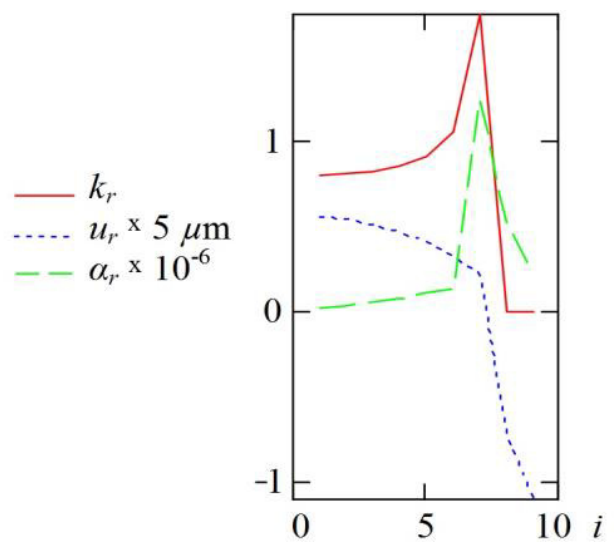

Fig. 7. Distribution of relative load concentration factors $\left(k_{r}\right)$, that occur in cables with numbers $i$, relative displacements $\left(u_{r}\right)$ and tangent of shear angles of elastic inter-cable layer $\left(\alpha_{r}\right)$ in a cross-section $x=0$ for $l_{1} \rightarrow \infty$

The effect of reduction of maximum tensile load concentration factors of cables is also observed in the case of breakage of the same cable in two cross-sections [20]. This effect can be used to increase the tractive ability of the belt with broken cables. For this, it is appropriate to artificially cut broken cables at a certain distance, or partially remove them. The latter may allow the removal of parts of cables in which the strings and wires lost their mutual connection, the connection formed by their twisting.

Note that the length used in studies above corresponds to mechanical characteristics of the belt RCB-3150. Indicators for this belt are indexed $b$. Indicate mechanical characteristics without a corresponding index. Considering the indicated designations, the obtained results can be extended to belts with other mechanical characteristics provided that they are proportional to the coefficient

$$
k=\sqrt{\frac{G k_{G}}{(t-d) E F} \frac{\left(t_{b}-d_{b}\right) E_{b} F_{b}}{G_{b} k_{G_{b}}}} .
$$

This is a consequence of the fact that the values of forces, displacements (1) are proportional to exponential functions. The argu- 
ments of functions are the product of values of a characteristic index $\beta_{n}$ and linear dimensions.

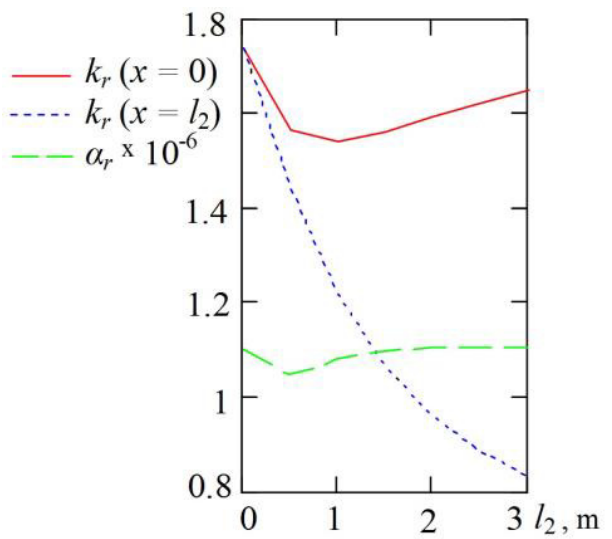

Fig. 8. Dependency of values of relative internal load concentration factors $k_{r}$ $(x=0)$ that occur in cables in a cross-section $x=0, k_{r}\left(x=l_{2}\right)$ in a cross-section $x=l_{2}$ and maximum shear angle tangents of elastic inter-cable layer $\left(\alpha_{r}\right)$ on the length of the second belt part when $l_{1} \rightarrow \infty$

\section{Belt with Irregularly Located Ruptures of Continuity of Cables}

Above we have considered the stress-strain state of a rope (belt) with regularly spaced cable breakages. During operation, the possible occurrence of a significant number of breakages of cables is possible. Their complex impact cannot be predicted. It must be determined considering the discrete values of numbers of broken cables and the location along the rope length. The number of breakages may be less than or equal to the number of cables in the rope. The most common case is the case of breakage of all cables. It corresponds to one of the possible schemes of butt-joint connection of rubber-cable belts. We will determine the displacements of cables, and the forces that occur in them using the expressions [19].

First, construct a model for the case of rupture of continuity of an arbitrary $j$-th rope (belt) of infinite length. Let the cross-section of the break have the coordinate $x=0$. Cut the rope into two parts. Give the parts numbers $1(x \leq 0)$ and $2(x \geq 0)$. As in previous cases, the part numbers are included in the indexes of quantities. The following conditions must be met in the cross-section $x=0$ 


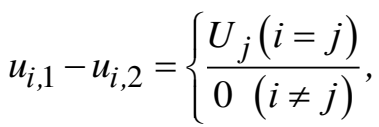

$$
\begin{aligned}
& p_{i, 1}-p_{i, 2}=0, \wedge p_{j, 1}=0 .
\end{aligned}
$$

When $x \rightarrow \pm \infty$

$$
\begin{aligned}
& u_{i, 1}-u_{i, 2} \rightarrow 0, \\
& p_{i, 1}=p_{i, 2} \rightarrow P .
\end{aligned}
$$

Consider the location symmetry of the cable breakage. Consider the part of the rope (belt) where $x \geq 0$ From condition (26) we have $A_{m}=0$.

The difference of displacements of edges of a broken cable is formulated as a sum of Fourier series

$$
u_{i, 1}-u_{i, 2}=\frac{2}{M} U_{j} \sum_{m=1}^{M-1} \cos \left(\mu_{m}(j-0.5)\right) \cos \left(\mu_{m}(i-0.5)\right)+\frac{1}{M} U_{j} \text {. }
$$

Displacements and internal loading forces on cables of the sample in accordance with [19] with arbitrary breakage will take the form

$$
\begin{gathered}
u_{i}=\frac{2}{M} U_{j} \sum_{m=1}^{M-1} \cos \left(\mu_{m}(j-0.5)\right) \cos \left(\mu_{m}(i-0.5)\right) e^{-\beta_{m} x}+\frac{1}{M} U_{j}+\frac{P}{E F} x, \\
p_{i}=\frac{2 E F}{M} U_{j} \sum_{m=1}^{M-1} \cos \left(\mu_{m}(j-0.5)\right) \cos \left(\mu_{m}(i-0.5)\right) e^{-\beta_{m} x_{3}} \beta_{m}+P .
\end{gathered}
$$

From (25) and (29) we determine the coefficient of proportionality and the expression for determining the distribution of forces between the cables

$$
U_{j}=-\frac{P M}{2 \sum_{m=1}^{M-1} \cos \left(\mu_{m}(j-0.5)\right)^{2} \beta_{m}} .
$$

The numbers of broken cables, the distances between the crosssections of gaps are combined into two sets $J$ and $L$. The crosssections with breakages are given the numbers $i$. We combine them into the set $I$. From the expression of the distribution of forces between the cables (29) we find the formula for the distribution of forces in the cross-sections of breakages of the cables 


$$
\begin{gathered}
p_{i, i \in I}=P \times \\
\left.\times \sum_{j \in J} \vartheta_{j} \frac{\sum_{m=1}^{M-1} \cos \left(\mu_{m}(j-0.5)\right) \cos \left(\mu_{m}(i-0.5)\right) e^{-\beta_{m}\left|l_{i}-l_{j}\right|_{\beta_{m}}}}{\sum_{m=1}^{M-1} \cos \left(\mu_{m}(j-0.5)\right)^{2} \beta_{m}}+1\right],
\end{gathered}
$$

where $\vartheta_{j}$ is the vector of proportionality coefficients.

The vector of proportionality coefficients is determined from the condition of absence of cable loads in the cross-sections of their breakages. Expression (27) in its expanded form is an algebraic system of equations. The solution of the system allows determining the vector of proportionality coefficients. Note that for an infinite distance between cable breakages, the components of the vector are equal to ones.

From expression (31) the displacements of cables in the crosssections of their breakages

$$
\begin{gathered}
u_{i, i \in I}= \\
=P \sum_{j \in J} \frac{\vartheta_{j}}{E F} \frac{\sum_{m=1}^{M-1} \cos \left(\mu_{m}(j-0.5)\right) \cos \left(\mu_{m}(i-0.5)\right) e^{-\beta_{m}\left|l_{i}-l_{j}\right|}}{\sum_{m=1}^{M-1} \cos ^{2}\left(\mu_{m}(j-0.5)\right) \beta_{m}} .
\end{gathered}
$$

Displacements of the cables (32) allow finding the maximum values of the tangents of shear angles of elastic material of rope shell in the local part, where the distance between points belonging to two adjacent cables in one cross-section is minimal.

Expressions (31) and (32) allow determining the SSS of a rope with any number of cables $M$ in the cross-sections of cable breakage. For example, consider a rope with five cables. All cables have gaps. The placement spacing of cross-sections with gaps is equal to $l$. The rope is loaded with a force that creates a single average load of cables (Fig. 9). 


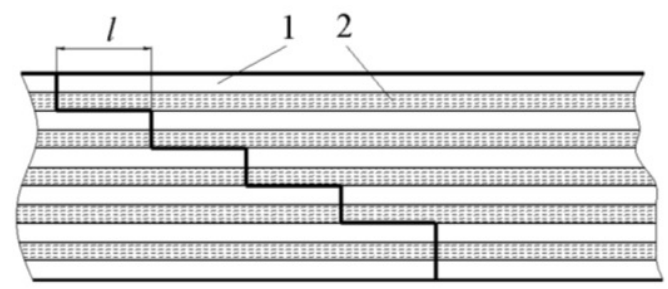

Fig. 9. Scheme of breakages of cables: 1 - layers of rubber, 2 - cables

Displacements, as well as loads of cables, are symmetrical with regard to the middle of a sample. The plane of symmetry of the belt coincides with the plane of symmetry of the middle cable when the number of cables is odd. The middle cable with an odd number of them will be considered the first one. In a rope with an even number of cables, the cables that are the closest to the middle of the rope are considered the first ones. Fig. 10 shows the ratios of coefficients of non-uniformity of force distribution in the extreme and middle cables for distances between the planes of breakages of $0.4 \mathrm{~m}, 0.5 \mathrm{~m}$ and $0.6 \mathrm{~m}$ and the variable number of cables in the sample.

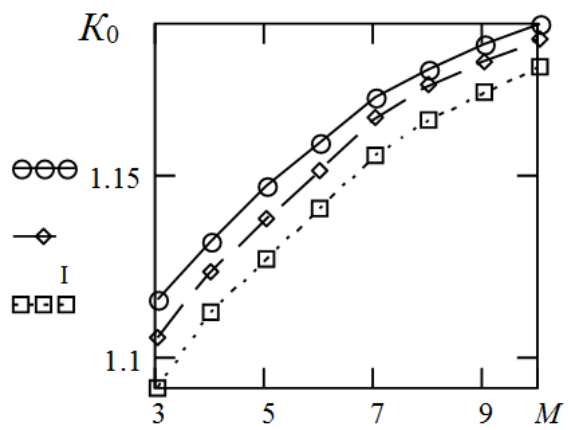

Fig. 10. Distribution of the ratio of the coefficients of non-uniformity of force distribution in the extreme and middle cables $K_{0}$ for the distances between the breakages $0.4 \mathrm{~m}, 0.5 \mathrm{~m}$ and $0.6 \mathrm{~m}$ and different numbers of cables $M$ : the upper curve is for $600 \mathrm{~mm}$, middle curve $-500 \mathrm{~mm}$, the lower curve - $400 \mathrm{~mm}$

The obtained dependencies show that the reduction of distances between the planes of discontinuities of cables leads to a decrease in the limits of changes in the coefficients of uneven distribution of forces between the cables. The outermost cables remain the most loaded. The coefficient of non-uniformity of force distribution for these cables is much higher than such coefficients for adjacent ca- 
bles. Further, consider the equal distances between the planes of the discontinuities of cables to be basic. Fig. 11 shows the ratios of the coefficients of non-uniformity of force distribution similar to the above for the case of reducing the lengths of the first and last crosssections of the sample relative to the basic by 20 and $30 \%$.

In the sample with an odd number of cables, the cross-section of the breakage of the middle cable coincides with the plane of symmetry. The sample of three cables has two areas located between the cross-sectional planes of the damaged cables. Changing the lengths of these areas is equal to changing the basic lengths. This case was from consideration. The graphical dependencies show that the reduction of lengths of the extreme areas leads to a decrease in the coefficients of unevenness of the loads of the extreme cables relative to the average. At the same time, it is even possible to exceed the maximum loads of the middle cables over the extreme ones. This pattern can be used to reduce the extreme loads of the cables of composite material with damage, such as in the butt-joints of the belts.

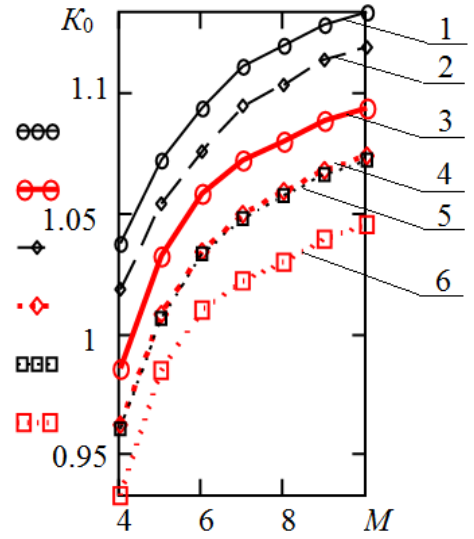

Fig. 11. Graph of the ratios of the coefficients of uneven force distribution in the extreme and middle cables $K_{0}$ for the base distances between the planes of the location of the gaps $400 \mathrm{~mm}, 500 \mathrm{~mm}$ and $600 \mathrm{~mm}$ and the different number of cables in the sample $M$. Denotations: $1-600 \mathrm{~mm}, 20 \%$ (black curve with circles); $2-500 \mathrm{~mm}, 20 \%$ (black dashed curve with rhombi); $3-600 \mathrm{~mm}, 30 \%$ (thicker red curve with circles); $4-500 \mathrm{~mm}, 30 \%$ (red dotted curve with rhombi); $5-400 \mathrm{~mm}, 20 \%$ (black dotted curve with squares); $6-400 \mathrm{~mm}, 30 \%$ (red dotted curve with squares)

According to the graphical dependencies of the displacement of 
the cables in the sample with breakages are different. Different displacements of cables lead to shear stresses in the inter-layers of elastic material. They are proportional to the tangents of the shear angles of elastic material between the cables. Expression (32) allows defining them in cross-sections of damage of cables

$$
=P \sum_{j \in J}\left(\begin{array}{c}
\gamma_{k, i \in I}= \\
\frac{\vartheta_{j}}{E F} \times \\
\times \frac{\sum_{m=1}^{M-1} \cos \left(\mu_{m}(j-0.5)\right)\left(\cos \left(\mu_{m}(k-0.5)\right)-\cos \left(\mu_{m}(k+0.5)\right)\right) e^{-\beta_{m}\left|l_{i}-l_{j}\right|}}{h \sum_{m=1}^{M-1} \cos ^{2}\left(\mu_{m}(j-0.5)\right) \beta_{m}}
\end{array}\right),
$$

$(1 \leq k<M)$.

Expressions of forces, displacements are the product of functions dependent on the cable number and the value of the $x$ coordinate. The last function in the obtained solution has decreased. The parameters of stress-strain state for cross-sections that do not coincide with the cross-sections of the damage are less important. The strength of the material is determined by the strength of the most loaded elements. In our case, these are the cables adjacent to the broken cable. Strength conditions of rubber-cable belt with random location of broken cables

$$
P\left[\sum_{j \in J} \vartheta_{j} \frac{\sum_{m=1}^{M-1} \cos \left(\mu_{m}(j-0.5)\right) \cos \left(\mu_{m}(j \pm 1-0.5)\right) e^{-\beta_{m}\left|l_{i}-l_{j}\right|_{\beta_{m}}}}{\sum_{m=1}^{M-1} \cos ^{2}\left(\mu_{m}(j-0.5)\right) \beta_{m}}+1\right] \leq[P],
$$

$$
\frac{G P}{t-d} \times
$$

$\times \sum_{j \in J}\left(\frac{\sum_{m=1}^{M-1}\left(\cos ^{2}\left(\mu_{m}(j-0,5)\right)-\cos \left(\mu_{m}(j \pm 1-0,5)\right) \cos \left(\mu_{m}(j-0,5)\right)\right) e^{-\beta_{m}\left|l_{i}-l_{j}\right|}}{h \sum_{m=1}^{M-1} \cos ^{2}\left(\mu_{m}(j-0,5)\right) \beta_{m}}\right) \leq[\tau]$.

Strength conditions must be fulfilled for both adjacent cables in all cross-sections of their breakage. In the general case, the total 
number of such conditions is four times the number of damages. Extreme cables have only one adjacent cable. The left parts of the strength conditions are given by symmetric functions. They are zero for $j=1 \wedge j=M$. In the case of damage to the extreme cables, the total number of conditions is less than twice the number of damaged extreme cables.

Fulfillment of the established conditions of strength allows making the reasonable decisions concerning conditions and admissibility of use of composite materials, in particular rubber-cable conveyor belts with the cables damaged in the course of operation, what provides safety of their use and utilizing their full resource.

\section{Conclusion}

Performed researches clarify the idea of the interaction mechanism of reinforcing elements in composite materials of layered structure with hard and soft layers. Boundary conditions significantly affect the stress-strain state of the belt.

An increase of the amount of partially removed cables leads to a nonlinear increase of relative load concentration factors and shears, provided that the amount of partially removed cables does not exceed the amount of unbroken cables. Absolute values of load concentration factors and shear values in the case of quantitative excess of partially removed cables over the amount of unbroken cables practically linearly increase with an increase of the amount of partially removed cables.

An increase of the amount of unbroken cables with the increase of a partially removed amount leads to a nonlinear increase of relative load concentration factors and shears. Absolute values of load concentration factors and shear values are nonlinearly reduced with an increasing amount of unbroken cables.

Values of maximum stresses in the belt with cables partially removed on a limited length depend on the amount of cables in the belt, the amount of partially removed cables, and the length of cable removal part.

The developed method allows determining the stress-strain state of the flat, reinforced with longitudinal tractive elements, belt of elastic material with various breakages of tractive elements, including the rupture of continuity and in the case of partial removal of elements under various boundary conditions. Method can be used in a control system of the conveyor belt during the belt life cycle. 
Further researches should be directed to a development of a method for determining the stress-strain state of the belt with damage, considering the design features of the conveyor base.

Using the methods of composite mechanics, a mathematical model of interaction of any number of soft and hard layers with the system of breakages to the latter is solved.

Analytical expressions of stress-strain state indicators of layered composite material with a given number of layers and local defects are obtained in a closed form.

The following is established by the analysis of the obtained solutions. The rupture of continuity of a cable leads to distortion of cross-sections of a belt. Damaged cable has larger displacements than the other ones. The uniform distribution of external loads between the cables is disturbed. The disturbance of the stress-strain state is localized both along the sample and along its thickness. Damage to the extreme cable leads to an increase in the tensile forces of the adjacent cable over $60 \%$ of the average load. The two cables closest to the damaged one are loaded with a force that exceeds their average total load by almost $80 \%$. The breakage of the middle cable leads to less disturbance.

The character of local stress disturbances also depends on the number of cables in the belt. As the total number of cables increases, the extreme forces occurring in the sample with the damaged cable decrease to almost constant values if the number of cables in a belt is not less than ten.

The linear formulation of the problem and the principle of superposition allows using the obtained dependencies in the case of applying force to one cable and fixing others.

The obtained solutions allow determining a stress-strain state of a rubber-cable belt and create conditions for making reasonable decisions regarding the conditions and admissibility of using rubber-cable belts with broken cables during operation, which ensures their operation safety and maximum utilization of their resource.

References

1. Dychkovskyi, R.O., Avdiushchenko, A.S., Falshtynskyi, V.S., \& Saik, P.B. (2013). On the issue of estimation of the coal mine extraction area economic efficiency. Naukovyi Visnyk Natsionalnoho Hirnychoho Universytetu, (4), 107-114.

2. Pivnyak, G., Dychkovskyi, R., Smirnov, A., \& Cherednichenko, Yu. (2013). Some aspects on the software simulation implementation in thin coal seams mining. Energy Efficiency Improvement of Geotechnical Systems, 1-11. 
3. Ilin, S.R., Samusia, V.I., Ilina, I.S., \& Ilina, S.S. (2016). Influence of dynamic processes in mine hoists on safety exploitation of shafts with broken geometry. Naukovyi Visnyk Natsionalnoho Hirnychoho Universitetu, (3), 42-47.

4. Kartavy, A.N., (2000). Justification of main parameters of steeply inclined conveyor with a clamping belt for quarries with large mineral extraction rate: PhD dissertation: 05.05.06.

5. Averchenkov, V.I., Davydov, S.V., Dunaev, V.P., \& Ivchenko, V.N. (2004). Conveyors with a suspended belt. Moskow.: Machine-building.

6. Pedchenko, O.S. (2007). Computational model of a suspended conveyor belt on a conveyor with a trajectory bended in a vertical plane. Mining informational and analytical bulletin, (1), 322-324.

7. Belmas, I.V., Kolosov, D.L., \& Kolosov, A.L. (2014). Study of stresseddeformed state of rubber-rope cable in the area of tubular transformation. Bulletin of PNRPU. Geology. Oil \& Gas Engineering \& Mining, (12), 48-55.

8. Kolosov, L.V., \& Bel'mas, I.V. (1981). Use of electrical models for investigating composites. Mechanics of Composite Materials, 17(1), 115-119.

9. Bel'mas, I.V. (1993). Stress state of rubber-rope tapes during their random damages. Problemy Prochnosti i Nadezhnos'ti Mashin, (6), 45-48.

10. Prushak, V.Ya. (2008). Numerical estimation of durability of connections of rubber-cable conveyor belt. Bulletin of BNTU, (1), 35-38.

11. Golikov, G.F., \& Ryabov, D.V. (2007). Criterions of estimation of operational quality of rubber-fabric conveyor belt and butt-joint connections. Mining informational and analytical bulletin, (10), 328-330.

12. Belmas, I.V., Perfilyeva, Yu.M., Daniyarov, N.A., \& Karsakova, A.Zh. (2013). Determination of stress-strain state of rubber-fabric belt with holes. Enbekteri university. University proceedings, (4), 60-64.

13. Gerasimov, G.K. (2006). Increase of operation reliability of rubber-cable belts of conveyor types 2LU120 and 2LTU120. Ugol, (9), 28-29.

14. Levchenya, Zh.B. (2004). Increase of reliability of butt-joint connections of conveyor belts at mining enterprises: $\mathrm{PhD}$ dissertation: 05.05.06.

15. Ishchenko R.V. (2013). Increase of reliability of high-wear parts of conveyor belts in air-salt mediums: PhD dissertation: 05.05.06.

16. Volohovskiy, V.Yu., Radin, V.P., \& Rudyak, M.B. (2010). Concentration of loads in cables and a bearing ability of rubber-cable conveyor belts with breakages. MPEI Vestnik, (5), 5-12.

17. Daria Zade S. (2013). Numerical method of determining effective characteristics of unidirectional reinforced composites. Bulletin NTU "KhPI", (58), 71-77.

18. Kolosov, D., Dolgov, O., \& Kolosov, A. (2013). The stress-strain state of the belt on a drum under compression by flat plates. Annual Scientific-Technical Collection - Mining of Mineral Deposits, 351-357.

19. Belmas, I.V., \& Kolosov, D.L. (2017). The stress-strain state of the flat rope caused by breaks of tractive elements and design of hoisting engine drum. Collection of research papers of national mining university, (50), 163-170.

20. Belmas, I.V., \& Bobylyova, I.T. (2012). Influence of cable breakages on a strength of a flat tractive element. Collection of the presentations of the participants of the 6th International Scientific and Methodical Conference, 88-91. 